\title{
Lesions of Rat Infralimbic Cortex Enhance Recovery and Reinstatement of an Appetitive Pavlovian Response
}

\author{
Sarah E.V. Rhodes and Simon Killcross ${ }^{1}$ \\ School of Psychology, Cardiff University, Cardiff, CF10 3YG, United Kingdom
}

\begin{abstract}
The prefrontal cortex (PFC) has a well-established role in the inhibition of inappropriate responding, and evidence suggests that the infralimbic (IL) region of the rat medial PFC (MPFC) may be involved in some aspects of extinction of conditioned fear. MPFC lesions including, but not those sparing the IL cortex increase spontaneous recovery of extinguished conditioned fear when tested $24 \mathrm{~h}$ after an initial extinction session. The current experiment extended these findings by use of appetitive rather than aversive conditioning. Ten IL-lesioned and 11 sham-operated rats were trained on a Pavlovian task in which a conditioned stimulus (CS) was followed by food pellets (the unconditioned stimulus or US). IL lesions had no effect on extinction of the conditioned response (CR, magazine entries) during the first extinction session. However, the level of spontaneous recovery between the first extinction session and a second, 24 h later, was increased in IL-lesioned rats relative to sham animals. In contrast, evidence of savings measured between the extinction sessions did not differ between groups. Furthermore, reinstatement of the CR following unsignaled delivery of the US was also increased in IL-lesioned rats.
\end{abstract}

Extinction reflects the decline in conditioned responding (CR) to a conditioned stimulus (CS) that once signaled the occurrence of reinforcement (the unconditioned stimulus, or US), but no longer does. It is generally accepted that this process involves the formation of new associations that override older associations that are no longer relevant in the extinction situation (Bouton 1994). In many cases, this new association is conceptualized as the development of new inhibitory links that reduce the likelihood of CRs (Konorski 1948, 1967; Rescorla 1993). Damage to the prefrontal cortex (PFC) frequently leads to an inability to inhibit responses that are no longer appropriate, for example, in the development of perseverative responses (Fuster 1997). Hence, the PFC may potentially serve as a source of inhibition during extinction, allowing new associations to override the ability of former associations to produce excitatory CRs. In rats, the medial PFC (MPFC) has been implicated in extinction of Pavlovianconditioned fear responses through the findings of several key studies.

Early work (Morgan et al. 1993; Morgan and LeDoux 1995) indicated that the ventral, but not the dorsal MPFC is involved in extinction of fear conditioning. They demonstrated that animals with lesions of this region took longer to extinguish a freezing CR to a tone CS that had originally been paired with footshock. However, the ventral MPFC lesions in this study encompassed portions of the infralimbic (IL), prelimbic, and medial orbital subregions of the MPFC. As well as being anatomically distinct, these areas are increasingly viewed as functionally distinct (Chudasama et al. 2003; Killcross and Coutureau 2003; Powell et al. 1994), raising the possibility that the effect found by Morgan and colleagues may have been due to disruption of a more specific subregion of the MPFC. Moreover, as there were only two extinction trials per session, this design could not dissociate between a deficit in the development of extinction within a session and the maintenance of extinction between sessions.

\section{'Corresponding author.}

E-MAIL KillcrossAS@cardiff.ac.uk; FAX 44-29-2087-4858.

Article and publication are at http://www.learnmem.org/cgi/doi/10.1101/ Im.79704.
This distinction is of considerable importance, as there are several phenomena associated with the maintenance of extinction, such as spontaneous recovery and reinstatement, which indicate that within and between-session extinction processes may differ. Spontaneous recovery refers to the finding that the expression of extinguished CRs recovers over time; despite successful extinction during one session, there is a transient restoration of the CR at the start of the next (for review, see Robbins 1990; Rescorla 2001). In reinstatement, the unsignaled presentation of a US after a learned response has been extinguished results in a similar transitory restoration of the conditioned response (Rescorla and Heth 1975).

The importance of this difference has been highlighted by recent work, which has further defined the role of the ventral MPFC in Pavlovian-conditioned fear extinction, as well as providing evidence that lesions restricted to the IL region lead to effects seen during extinction. Quirk et al. (2000) demonstrated that lesions of the IL cortex had no effect on extinction of a conditioned fear response within a session. However, IL lesions did result in increased spontaneous recovery relative to shams at the start of a subsequent extinction session, $24 \mathrm{~h}$ after the first. The level of recovery of CRs shown by IL-lesioned rats was similar to the initial level of CRs observed in extinction-naive rats which had not been subject to the initial extinction training session. Nevertheless, despite equivalent responding at the start of the session, IL-lesioned animals extinguished responding at a greater rate than the extinction-naive group, showing savings that indicated normal expression of extinction between sessions. These results demonstrate that on the second extinction session, the IL-lesioned animals had clearly retained some aspects of the extinction training (hence, demonstrating savings), but this extinction training failed to dominate performance at the start of the test session, manifest as increased recovery.

As far as we are aware, research in this area to date has investigated the role of the IL in extinction of conditioned fear. The role of this region in extinction of appetitive Pavlovian conditioning has not yet been considered; the current experiment addresses this issue. After seven sessions of appetitive Pavlovian conditioning to an auditory or visual CS, animals underwent a 
single session of extinction. After $24 \mathrm{~h}$, they were tested under extinction for the occurence of spontaneous recovery, and then reinstatement of responding following unsignaled presentations of the reward. This test sequence was repeated three times.

\section{RESULTS}

\section{Histological Analysis}

Figure 1 illustrates the location and extent of the IL lesions; placement was verified with reference to Paxinos and Watson (1997). As a result of examination of the stained sections under a light microscope, two lesioned rats were excluded from statistical analysis because their lesions were not considered large enough, and one further lesioned rat was removed because it had extra cortical damage due to an infection. Figure 2 shows photomicrographs of a coronal section of the MPFC region of both a shamand an IL-lesioned rat. Post histology, the final number of rats of each group was $10 \mathrm{IL}-$-lesioned and 11 Sham-lesioned rats.

\section{Behavioral Results}

Preliminary analyses of variance conducted on the recovery and reinstatement data using a $2 \times 2 \times 3 \times 2$ mixed-ANOVA with factors of lesion group (IL or Sham), modality of CS (auditory or visual), test number (repetitions 1-3), and test type (recovery or reinstatement). This indicated that there was a main effect of modality $\left[F_{(1,19)}=12.78, P<0.002\right]$ with greater overall responding to the auditory CS. However modality did not interact with lesion group or test type (largest $F_{(1,19)}=3.21, P>0.05$ ). There-
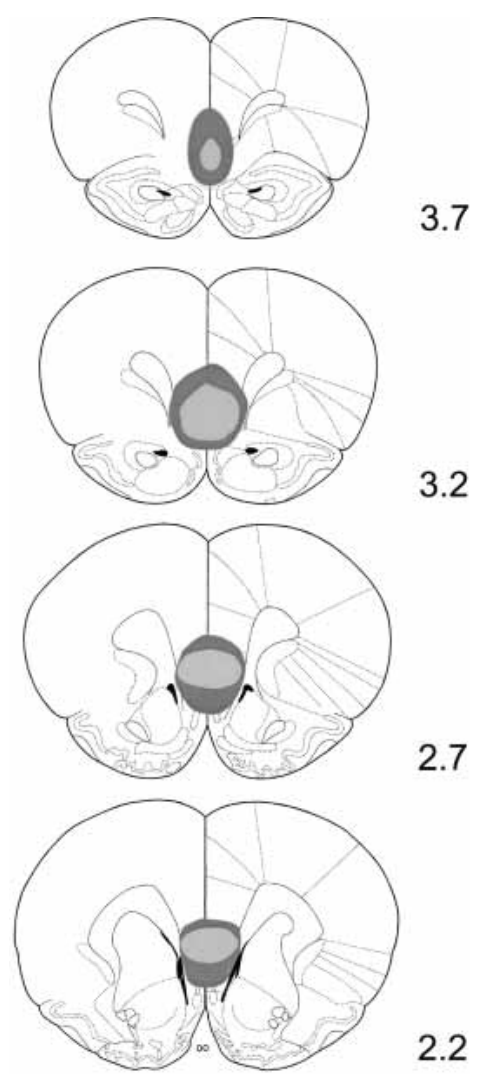

Figure 1 Schematic representation of the IL lesion showing the maximum (dark gray) and minimum (light gray) extent of cell damage and gliosis due to ibotenic acid infusions. Reconstructions were made on the basis of coronal sections (Paxinos and Watson 1997) at appropriate distances (millimeter) anterior to bregma.
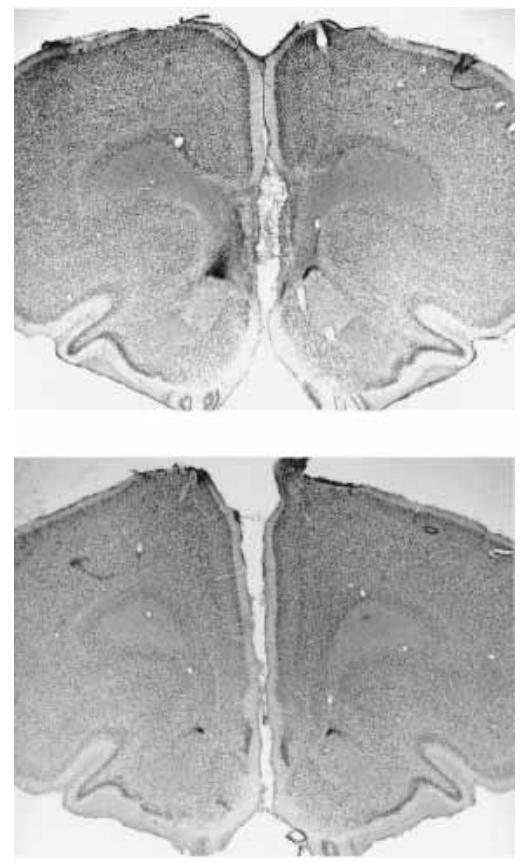

Figure 2 Photomicrograph of a bilateral section through an IL-lesioned brain (top) and a sham-lesioned brain (bottom) at $\sim 3.2 \mathrm{~mm}$ anterior to bregma.

fore, the data were collapsed across the two modalities for subsequent analyses.

\section{Acquisition}

All animals successfully acquired the magazine entry CR during appetitive Pavlovian conditioning, and there was no difference between sham and lesioned groups in the rate of acquisition. A $2 \times 7$ mixed-ANOVA with factors of lesion group (sham, IL) and session (1-7) revealed a main effect of session $\left(F_{(6,114)}=31.9\right.$, $P<0.001)$ but no main effect of lesion group and no group $\mathrm{x}$ session interaction (both $F s<1$ ). Furthermore, there was no difference in the level of responding on the final acquisition session (mean CS-preCS magazine entries per minute: $\mathrm{IL}=21.97$ and Sham $=20.59,[\mathrm{t}(19)=0.42 P>0.05])$.

\section{Within-Session Extinction}

To establish whether IL lesions disrupted within-session extinction, the trial-by-trial performance of both groups during the first extinction session was considered (Fig. 3). These data were analyzed in a $2 \times 16$ mixed-ANOVA with factors of lesion group and trial that revealed no difference between groups in the rate or extent of extinction. There was a significant main effect of trial $\left(F_{(15,285)}=18.89, P<0.001\right)$, indicative of extinction, but no trial $\mathrm{x}$ lesion group interaction and no main effect of lesion group (both Fs < 1).

\section{Spontaneous Recovery and Reinstatement}

There was evidence for spontaneous recovery between sessions, and reinstatement of responding following unsignaled presentations of the US in both groups. IL lesions resulted in a greater increase in CRs in both cases compared with sham animals. ILlesioned rats showed recovery to almost $100 \%$ of the preextinction CR. Figure 4 shows the decline in performance between the initial and subsequent extinction sessions; recovery and reinstatement on the first trial of these sessions is highlighted in the filled (sham) and open (IL) bars.

\section{Learning \& Memory}

www.learnmem.org 


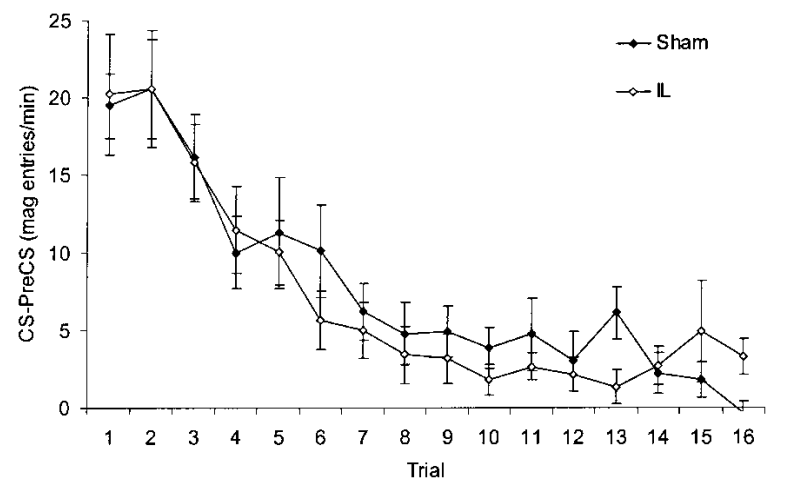

Figure 3 Mean CRs (mean CS-preCS magazine entries per minute \pm SEM) during trials across the first session of extinction.

As these assessments examine the increase in responding from the end of one extinction session to the start of the next, the increase in CRs from the final block of four trials of one session to the first trial of the next was calculated. The mean increase in responding (CS-PreCS magazine entries per minute) over the three test days was as follows: Sham $=13.0$ and IL $=19.0$ for recovery (i.e., mean increase from extinction sessions 1-2, 3-4, and 5-6) and Sham $=3.9$ and $\mathrm{IL}=12.1$ for reinstatement (i.e., mean increase from extinction sessions $2-3,4-5$, and 6-7).

A $2 \times 3 \times 2$ mixed-ANOVA with factors of lesion group (IL, Sham), test number (repetitions 1-3) and test type (recovery or reinstatement) was carried out on these data. Most importantly, this revealed a significant main effect of group $\left(F_{(1,19)}=10.6\right.$, $P<0.005)$ but no group $\mathrm{x}$ test type interaction $(F<1)$ supporting the claim that there is greater recovery and reinstatement in ILlesioned animals. There was also a main effect of test type $\left(F_{(1,19)}=14.2, P>0.001\right)$, as there was greater spontaneous recovery than reinstatement in all groups. There was no main effect of test number, nor any interaction of group with either test number, test type, or both (maximum $F_{(2,38)}=1.6,>0.05$ ).

\section{Savings}

A savings analysis was carried out in order to establish whether there was any difference between IL-lesioned animals and sham animals in the progression of extinction across multiple extinction sessions. A trial-by-trial analysis was conducted to compare

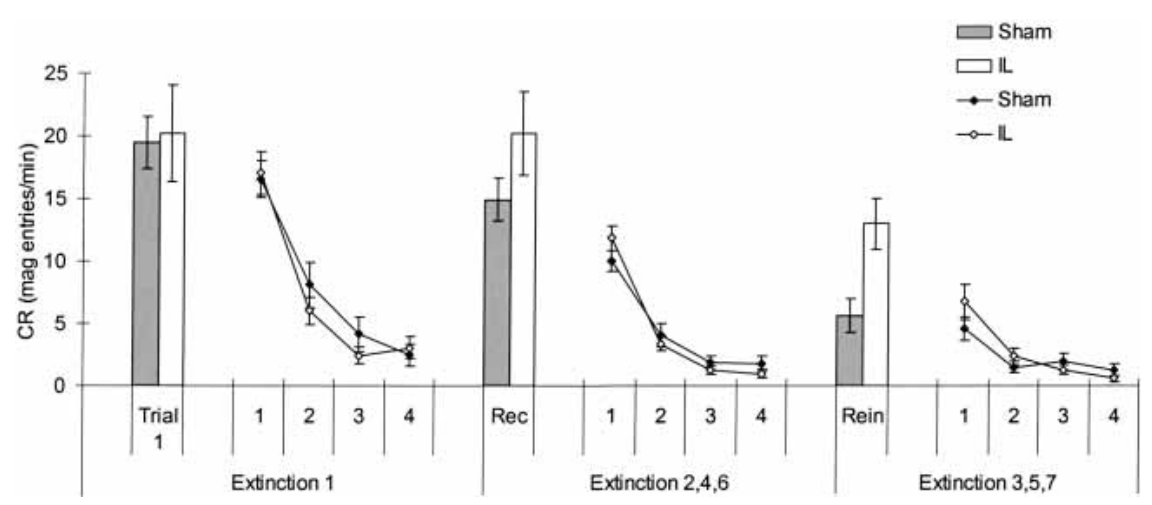

Figure 4 Mean CRs (mean CS-preCS magazine entries per minute \pm SEM) across extinction sessions. Lines represent responding in blocks of four trials across the first extinction session and the recovery and reinstatement sessions. The first bar (Trial 1) represents responding on the first trial of the first extinction session. The second and third bars represent the mean responding on the first trial of extinction sessions testing for spontaneous recovery (Rec; i.e., sessions 2, 4, and 6) and reinstatement (Rein; i.e., sessions 3, 5, and 7), respectively. performance on the first extinction session with the average performance on the first extinction session of the subsequent test days. A $2 \times 2 \times 16$ mixed-ANOVA with factors of lesion group (IL, Sham), session (first extinction or subsequent extinction), and trial (1-16) was carried out. There was a main effect of trial $\left(F_{(15,285)}=36.2, P<0.001\right)$ and a session $\mathrm{x}$ trial interaction $\left(F_{(15,285)}=2.9, P<0.001\right)$, indicating, respectively, that extinction did occur in all sessions, but that there were savings from the first extinction session to subsequent sessions. Furthermore, there was no main effect or interaction involving group $(F s<1)$, indicating that savings in both groups were equivalent. Therefore, although IL lesions result in increased recovery and reinstatement of a CR at the start of test sessions, this increase in responding above that of sham animals is not maintained across the session.

\section{DISCUSSION}

The current study investigated the role of the IL MPFC in acquisition and maintenance of extinction of an appetitive Pavlovian CR. Both sham and IL-lesioned animals displayed spontaneous recovery of the CR when tested $24 \mathrm{~h}$ after the initial extinction, and reinstatement of the CR following unsignaled presentations of the reinforcer. Both of these effects were significantly greater in IL-lesioned animals. We found no effect of IL lesions on extinction within the initial extinction session nor on the savings from one extinction session to the next.

These findings parallel previous demonstrations of the role of the MPFC in extinction of an aversive Pavlovian response, extending the research to the appetitive domain. As in the current experiment, a study by Quirk et al. (2000) also found no effect of IL lesions on within-session extinction, or savings between sessions (but see Santini et al. 2003); but IL lesions did produce a significant increase in spontaneous recovery. However, although there was evidence for a trend toward an increase in reinstatement in IL-lesioned animals in their study, this effect was not significant. The difference in the results between studies could be because reinstatement in aversive and appetitive paradigms may be qualitatively different, although it may equally be a simple, parameter-dependent effect. Although our results support and extend evidence for the involvement of the IL MPFC in extinction, they do not address the possible role of other MPFC subregions. This issue has, however, been directly investigated by previous studies (Quirk et al. 2000; Milad and Quirk 2002), the results of which suggest that the MPFC's role in extinction is solely due to functioning of the IL subregion. Similarly, although we did not include control groups to show that the reinstatement effect was due to unpaired US presentations rather than the brief (10 min) delay during which the USs were presented, similar delays between CS presentations in appetitive conditioning have not by themselves resulted in reinstatement of responding, but rather a progressive decline in CRs due to continued extinction (e.g., experiment 1; Bouton and Peck 1989).

Quirk et al. (2000) postulated that the IL MPFC was required for long-term storage of extinction learning. However, consideration of the current findings allows us to conclude that the IL MPFC is unlikely to be necessary for storage/retention of extinction learning, as we found no IL-lesion deficit in the savings (the increased rate of within-session extinction that develops 
with multiple extinction sessions) between subsequent sessions. Furthermore, the IL MPFC is not essential for acquisition of extinction learning as IL-lesioned animals showed no deficit on the initial extinction sessions. However, the IL MPFC is involved in the rapid expression of extinction at the start of extinction sessions. Support for the notion that the IL MPFC is not required for acquisition or retention of extinction per se, but that it is involved in some aspect of expression of extinction at a later date, has also been shown with electrophysiological recording data. Milad and Quirk (2002) showed that the IL region is not active during an initial extinction session, but is active during subsequent extinction sessions, particularly at the start of a session. Other studies have also found an increase in prefrontal activity during the recall of extinction in both mice (Barrett et al. 2003) and humans (Schreurs et al. 2001). Furthermore the ability of IL-lesioned rats in the current study to express extinction normally as each extinction session progressed, implies that these animals have no working or short-term memory deficit, a finding also supported by previous studies (Delatour and Gisquet-Verrier 1996; Ragozzino et al. 1999).

Extinction learning is no longer viewed as an associative loss as predicted by traditional learning theories (Rescorla and Wagner 1972; Mackintosh 1974). Currently, a common view is that extinction involves the formation of new CS-US inhibitory associations (or excitatory CS-noUS associations), which counteract original excitatory CS-US learning (Konorski 1948, 1967; Rescorla 1993). Extinction can be conceptualized as producing new learning that competes with the original conditioning, resulting in a behavioral or associative conflict that must be resolved. Therefore, despite successful extinction training, under certain conditions (such as following the passage of time or a reminder of the US), the original excitatory associations "win-out" over the inhibitory associations acquired in extinction, and the extinguished CR is performed (leading to phenomena such as spontaneous recovery and reinstatement).

One simple model of extinction suggesting that spontaneous recovery and reinstatement may arise from a common underlying mechanism is based around the importance of contextual cues (Bouton 1993, 1994). It is well established that extinction is context dependent. According to Bouton's model, the impact of contextual cues on extinction occurs due to the ability of these cues to modulate the new learning that occurs in extinction. Hence, the expression of newly developed CS-US inhibitory associations, or CS-noUS associations, is under the influence of contextual cues to a far greater degree than the original CS-US excitatory associations (Bouton 1994).

According to this account, spontaneous recovery occurs as the interval between preliminary extinction and subsequent testing produces a change in contextual cues as a result of the passage of time (for example, due to a change in the internal state of the animal). As these contextual cues available at test differ from those present during extinction, CRs recover transiently at test due to expression of the more contextually independent excitatory CS-US associations, rather than the contextually modulated inhibitory CS-US associations formed in extinction. As the test session proceeds, the occurrence of extinction trials re-establishes the extinction context, and performance comes to reflect previous and ongoing extinction learning. Similarly, in reinstatement, the presentation of the US before test ensures that the test context is more similar to the original conditioning context (in which reinforcement occurred) than the extinction context (in which no reinforcement occurred).

Within this framework, lesions of the IL cortex may be seen as interfering with this contextual modulation of extinction learning. If lesions of the IL cortex alter the process whereby contextual cues boost retrieval of newly acquired extinction as- sociations, then both recovery and reinstatement will be increased as the dominance of performance by prior excitatory CSUS associations will be increased. However, it is crucial to distinguish between the role played by context in providing cues for behavior at the start of a test session, and the role played by alternative cues that arise within the test session that may also modulate performance. Hence, modulation of performance at the very start of the session depends on the ability to transfer contextual modulation of associations from one session to the next. In contrast, cues within the test extinction session itself (such as the continuing absence of the US) can give rise to within-session modulation of associations. Lesions of the IL cortex influence the former process, but not the latter. Furthermore, if it is assumed that with each extinction session, the associations formed in extinction become stronger, until eventually they become strong enough to override the excitatory CS-US association without contextual modulation (i.e., they become the dominant associations), then both recovery and reinstatement would decline in magnitude with repeated testing, and the influence of IL lesions would also decrease (Lebron et al. 2003).

Although the results are straightforwardly interpreted as a deficit in transfer of contextual modulation between sessions, but not of extinction learning or contextual modulation (e.g., by within-session cues) of this learning per se, the behavioral effects of the lesion may also be conceptualized as reflecting an increased level of contextual control over newly acquired extinction associations. By this account, lesions of the IL cortex produce their effects by increasing the context dependency of extinction. Hence, any normal recovery or reinstatement due to a shift in contextual cues will be enhanced, as the effective shift away from the extinction context is greater. Once again, within-session extinction cues (which may even have a greater impact in lesioned animals) can produce normal extinction performance as the session continues. This interpretation is not necessarily opposed to the suggestion above; the failure to transfer contextual control of extinction associations between sessions could be due to an increased dependence on the precise contextual cues present for extinction.

The notion that new associations formed during a second phase of training come to be modulated by contextual cues is not restricted to extinction; many other conditioning procedures involve the development of new associations that must override existing ones (Bouton 1993). Reversal learning and counterconditioning paradigms reflect situations in which a previously learned, but now inappropriate response to a CS must be overridden in favor of a newer, currently relevant response. We have found that selective IL lesions disrupt reversal learning in an appetitive Pavlovian procedure (S. Rhodes, unpubl.).

In summary, we have found an increase in spontaneous recovery and reinstatement following lesions of the IL MPFC. Consideration of the theoretical underpinnings of these phenomena leads us to suggest that IL lesions may interfere with the manner whereby contextual cues modulate inhibitory associations in extinction, but do not influence the formation or retention of these inhibitory associations per se. Although perhaps counterintuitive, this hypothesis is not at odds with the suggestion that IL lesions could increase the contextual specificity of extinction learning.

\section{MATERIALS AND METHODS}

\section{Subjects}

Twenty-four male Lister Hooded rats (Harlan) were housed in pairs in a temperature-controlled room on a 14-h light:10-h dark cycle; all testing occurred at a regular time during the light pe-

\section{Learning \& Memory}


riod. The animals had free access to water, but were food deprived and maintained at $85 \%$ of matched free-feeding body weight throughout the experiment. Their weights before behavioral testing were 321-415 g $(\underline{\mathrm{M}}=359 \mathrm{~g}, \underline{\mathrm{SD}}=21.25)$. All procedures involving animals and their care conformed to the institutional guidelines that comply with national [Animals (Scientific Procedures) Act 1986] and international (Directive 86-609, November 24, 1986, European Community) laws and policies.

\section{Surgical Procedures}

Animals received bilateral excitotoxic lesions of the IL cortex, or sham surgery. The rats were anesthetized with isoflurane and then placed in a Kopf stereotaxic frame (Kopf Instruments) in a flat skull position with the incisor bar set at $-3.3 \mathrm{~mm}$. Lesions of the IL cortex were made $(n=13)$ by infusing $0.15 \mu \mathrm{L}$ ibotenic acid (63 mM in PBS; Biosearch Technologies Inc.) through a syringe (SGE International Pty Ltd) with a rounded tip, at the following coordinates from bregma: $\mathrm{AP}=2.6 \mathrm{~mm} ; \mathrm{ML} \pm 0.6 \mathrm{~mm}$, $\mathrm{VM}-5.4 \mathrm{~mm}$. Control rats $(n=11)$ received an identical surgical procedure with the exception of infusion of the neurotoxin.

All subjects recovered for a period of over $15 \mathrm{~d}$ after surgery with ad lib access to food and water, after which the food deprivation schedule was maintained for $7 \mathrm{~d}$ prior to the start of behavioral procedures.

\section{Apparatus}

Eight standard operant chambers $(30 \times 24 \times 22 \mathrm{~cm}$; MedAssociates), each consisting of a metal frame with clear Perspex walls, housed in a light- and sound-attenuating box, were used for behavioral testing. The box was fitted with a fan for ventilation and to mask extraneous noise. Rats were placed into the chamber via a Perspex door in the front. The floor of each chamber consisted of 19, 5-mm diameter, stainless-steel bars spaced $1.1 \mathrm{~cm}$ apart. The chambers were illuminated by a diffuse, upward-shining 4.2-W house light fixed on the left-hand wall of the chamber. A food magazine was built into the right-hand wall and entry was detected by the disruption of an infrared beam between the mouth of the magazine. Pellets ( $45 \mathrm{mg}$, PJ Noyes) were delivered into the magazine from a pellet dispenser mounted outside of the chamber on the wall containing the magazine. Two CSs were available in the chambers. One comprised illumination of two stimulus lights $(2.8-\mathrm{W})$, which were mounted on the right-hand wall, either side, and above the food magazine. The other CS was the presentation of a $3-\mathrm{kHz}$ tone $(96 \mathrm{~dB})$ produced by an in-house audio signal generator and delivered through a wall-mounted speaker in the top, left-hand corner of the chamber. A Dantum II/B computer equipped with Med-Pc software (Med-Associates) was used to control the experiment and record the data.

\section{Behavioral Procedures}

All rats underwent one session of magazine training lasting $30 \mathrm{~min}$, during which the house light was on, and all animals received pellets on a variable time 60 -sec schedule. Following this, an appetitive Pavlovian conditioning procedure was used, in which rats came to approach the food magazine during the presentation of a CS that predicted delivery of food pellet rewards. The CR measured during all stages of the experiment was magazine approach. Acquisition sessions lasted 29 min with 12, 15-sec CSs flanked by a variable ITI of about $2 \mathrm{~min}$; a single food pellet reward was delivered at the end of each CS. After seven acquisition sessions, rats had acquired robust magazine approach CRs and had reached asymptote in responding. Following the final acquisition session, rats were returned to their home cage for $1 \mathrm{~h}$, and then given one session of extinction training. This extinction session lasted $38 \mathrm{~min}$, with 16, 15-sec CSs flanked by a variable ITI of about 2 min; no pellets were delivered following the CS.

On the following day, the animals received a further session of extinction (as above) in order to test for spontaneous recovery. Following this test session, the animals remained in their test boxes for $5 \mathrm{~min}$, and then received five unsignaled food pellets over a 30-sec period. They remained in their boxes for a further $5 \mathrm{~min}$, and then received a further extinction test to examine reinstatement of responding. This entire test day procedure (sequential test sessions for recovery and reinstatement on the same day) was repeated twice more, with a gap of $4 \mathrm{~d}$ between test days, so that the test days occurred 1,6 , and $11 \mathrm{~d}$ after the initial extinction training. There was no retraining between test days.

The entire procedure outlined above, involving acquisition and extinction of Pavlovian conditioning, followed by tests for spontaneous recovery and reinstatement, was carried out twice within the same subjects, using the different CSs. Hence, rats were trained in turn with the auditory CS (tone), and the visual CS (stimulus lights).

\section{Histology}

After behavioral testing, animals received a lethal dose of sodium pentobarbitone and were perfused transcardially with $0.9 \%$ saline, followed by $10 \%$ formal saline. The brains were then removed and left to dehydrate in $25 \%$ sucrose solution for a minimum of $2 \mathrm{~d}$. Coronal sections $(60-\mu \mathrm{m}$ thick) of the brains were cut using a freezing microtome $\left(-20^{\circ} \mathrm{C}\right)$, and every second section was mounted onto gelatin-coated slides. These were dried at room temperature for $24 \mathrm{~h}$ before staining with cresyl violet, followed by the addition of a coverslip in DPX. These sections were used to determine the location and extent of the lesions.

\section{Data Analysis}

The dependent variable recorded was magazine entries per minute; to reduce variability due to differences in baseline responding by different animals, a CS-preCS measurement was used. The preCS value paired with a particular CS value was based on responding during the ITI period preceding that CS period. Data were subjected to analysis of variance (ANOVA); where interactions between factors were significant, simple effects analyses were conducted.

\section{ACKNOWLEDGMENTS}

The research was supported by a MRC studentship to S.R. and an MRC Career Establishment Grant to S.K. (Grant ref. G9805345). We thank Mark Bouton for preliminary discussions of the data presented in this article.

\section{REFERENCES}

Barrett, D., Shumake, J., Jones, D., and Gonzalez-Lima, F. 2003. Metabolic mapping of mouse brain activity after extinction of a conditioned emotional response. J. Neurosci. 23: 5740-5749.

Bouton, M. 1993. Context, time and memory retrieval in the interference paradigms of Pavlovian learning. Psychol. Bull. 1141: 80-99.

. 1994. Conditioning, remembering and forgetting. J. Expt. Psychol.: Anim. Behav. Proc. 20: 219-231.

Bouton, M. and Peck, C. 1989. Context effects on conditioning, extinction and reinstatement in an appetitive conditioning preparation. Anim. Learn. Behav. 17: 188-198.

Chudasama, Y., Passetti, F., Rhodes, S., Lopian, D., Desai, A., and Robbins, T. 2003. Dissociable aspects of performance on the 5-choice serial reaction time task following lesions of the dorsal anterior cingulate, infralimbic and orbitofrontal cortex in the rat: Differential effects on selectivity, impulsivity and compulsivity. Behav. Brain Res. 146: $105-119$.

Delatour, B. and Gisquet-Verrier, P. 1996. Prelimbic cortex specific lesions disrupt delayed-variable response tasks in the rat. Behav. Neurosci. 110: 1282-1298.

Fuster, J. 1997. The prefrontal cortex: Anatomy, physiology, and neuropsychology of the frontal lobe. 3rd ed., Lippincott-Raven, New York.

Killcross, A. and Coutureau, E. 2003. Coordination of actions and habits in the medial prefrontal cortex of rats. Cereb. Cortex 13: 400-408.

Konorski, J. 1948. Conditioned reflexes and neuron organisation. Cambridge University Press, New York.

1967. Integrative activity of the brain. University of Chicago Press, Chicago, IL.

Lebron, K., Milad, M., and Quirk, G. 2003. Rats with lesions of the ventromedial prefrontal cortex can eventually recall extinction, with 
additional training. Program No. 199.52003 Abstract Viewer/Itinerary Planner, Society of Neuroscience, Washington, DC. (Online).

Mackintosh, N. 1974. The psychology of animal learning. Academic Press, London.

Milad, M. and Quirk, G. 2002. Neurones in medial prefrontal cortex signal memory for fear extinction. Nature 420: 70-74.

Morgan, M. and LeDoux, J. 1995. Differential contribution of dorsal and ventral medial prefrontal cortex to the acquisition and extinction of conditioned fear in rats. Behav. Neurosci. 109: 681-688.

Morgan, M., Romanski, L., and LeDoux, J. 1993. Extinction of emotional learning: Contribution of medial prefrontal cortex. Neurosci. Lett. 163: 109-113.

Paxinos, G. and Watson, C. 1997. The rat brain in stereotaxic coordinates. 3rd ed., Academic Press, London.

Powell, D., Watson, K., and Maxwell, B. 1994. Involvement of subdivisions of medial prefrontal cortex in learned cardiac adjustments in rabbits. Behav. Neurosci. 108: 294-307.

Quirk, G., Russo, G., Barron, J., and Lebron, K. 2000. The role of ventromedial prefrontal cortex in the recovery of extinguished fear. J. Neurosci. 20: 6225-6231.

Ragozzino, M., Detrick, S., and Kesner, R. 1999. Involvement of the prelimbic-infralimbic areas of the rodent prefrontal cortex in behavioural flexibility for place and response learning. J. Neurosci.
19: 4585-4594.

Rescorla, R. 1993. Inhibitory associations between S and R in extinction. Anim. Learn. Behav. 21: 327-336.

. 2001. Retraining of extinguished Pavlovian stimuli. J. Expt. Psychol.: Anim. Behav. Proc. 27: 115-124.

Rescorla, R. and Heth, C. 1975. Reinstatement of fear to an extinguished conditioned stimulus. J. Expt. Psychol.: Anim. Behav. Proc. 1: 88-96.

Rescorla, R. and Wagner, A. 1972. A theory of Pavlovian conditioning: Variations in the effectiveness of reinforcement and nonreinforcement. In Classical conditioning II: Current research and theory. (ed. A. Black), pp. 64-99. Appleton-Century-Crofts, New York.

Robbins, S. 1990. Mechanisms underlying spontaneous recovery in autoshaping. J. Expt. Psychol.: Anim. Behav. Proc. 16: 235-249.

Santini, E., Burgos-Robles, A., and Quirk, G. 2003. Protein synthesis in the medial prefrontal cortex is required for consolidation of extiction memory. Program No. 199.4 2003 Abstract Viewer/Itinerary Planner. Society of Neuroscience, Washington, DC. (Online).

Schreurs, B., Bahro, M., Molchan, S., Sunderland, T., and McIntosh, A. 2001. Interactions of prefrontal cortex during eyeblink conditioning as a function of age. Neurobiol. Ageing 22: 237-246.

Received April 8, 2004; accepted in revised form June 23, 2004.

\section{Learning \& Memory}




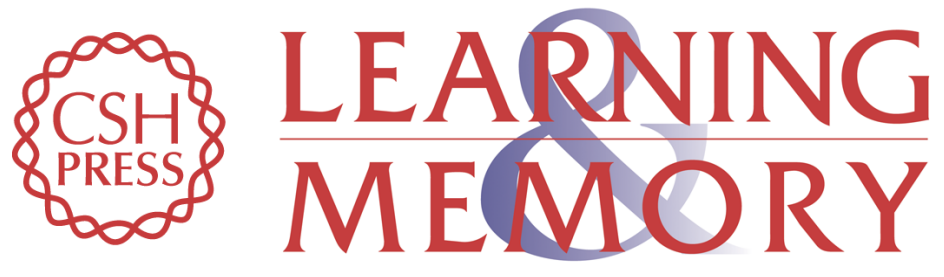

\section{Lesions of Rat Infralimbic Cortex Enhance Recovery and Reinstatement of an Appetitive Pavlovian Response}

Sarah E.V. Rhodes and Simon Killcross

Learn. Mem. 2004, 11:

Access the most recent version at doi:10.1101//m.79704

References This article cites 15 articles, 3 of which can be accessed free at: http://learnmem.cshlp.org/content/11/5/611.full.html\#ref-list-1

License

Email Alerting

Receive free email alerts when new articles cite this article - sign up in the box at the Service top right corner of the article or click here. 